\title{
Image Compression Using Quaternion Wavelet Transform
}

\author{
${ }^{* 1}$ C. Madhu, ${ }^{2}$ E. Anant Shankar \\ ${ }^{1,2}$ Department of ECE, SV College of Engineering, Tirupati, India
}

IEmail: cherukulamadhu@gmail.com

\section{Received: 06th October 2017 Accepted: 14th November 2017, Published: 31st December 2017}

\begin{abstract}
:
The target of image compression is to diminish insignificance and repetition of the picture information keeping in mind the end goal to have the capacity to store or transmit information in a proficient manner. The change from the cine film to advanced strategies for picture trade and authentic is basically persuaded by the simplicity and adaptability of dealing with computerized picture data rather than the film media. While setting up this progression and creating guidelines for computerized picture correspondence, one need to ensure that additionally the picture quality is kept up or made strides. This paper proposes wavelet based compression by taking color flow in to consideration, so that the proposed wavelet transform will give priority to the phase components.
\end{abstract}

\section{KEYWORDS:}

DWT, Complex Wavelet Transform, Quaternion Wavelet Transform, Compression Ratio, Image Compression, Peak Signal to Noise Ratio, Quaternion Algebra

\section{INTRODUCTION}

One of the intense scientific apparatuses in the area of signal and image processingis discrete wavelet Transform (DWT) [1], it additionally endures with few cons. For example, on the off chance that we are taking stage in to thought, this DWT won't work better since it doesn't contain any nonexistent terms like ordinary Fourier transform. Keeping in mind the end goal to get this favorable position and to maintain a strategic distance from few the cons from the DWT one can go for complex wavelet Transform [2]. However, in the event that one needs to break down the picture as far as its color flow or intensity flow [3] complex wavelet transform neglects to accomplish this and create signal phase equivocalness when spoken to two-dimensional picture's components. Thus, to stay away from every one of these issues in picture examination this paper proposes another multiscale analysis tool called "Quaternion Wavelet Transform" QWT, which is a current change of standard wavelets. It relies upon on the Hilbert -2D transform speculation, which has hard circulated invariance and may well beat the above downsides [4-6].

At show off, quaternion wavelet observe is isolated into branches, one depends on quaternion numerical capability multiresolution exam speculation of quaternion wavelet, utilising a solitary tree structure,
The maximum punctual in 1994, quaternion wavelet frame thought was given by Mitrea[8]; in 2001, Traversoni utilized true wavelet alternate and complex wavelet trade by using quaternion Haar piece and proposed discrete quaternion wavelet transfom [9] and gave some applications in picture coping with; in 2004, $\mathrm{He}$ and $\mathrm{Yu}$ applied grid esteem paintings multiresolution studies shape for lower back to again quaternion wavelet alternate [10] and gave some houses; methodicallly improved the lower returned to returned wavelet transform idea to successive quaternion wavelet concept [12], and established the duplicate speculation and constant quaternion wavelet important residences. Be that as it can, these are for the maximum element the thoughts and properties of development, due to the fact its channels' shape and utilization are demanding situations, it has now not received any floor in utility at display. Another department depends on Bulow quaternion diagnostic flag [13]; Utilizing actual channel and double tree shape to perform the quaternion wavelet transform. CWT simply has one stage, even as QWT can deliver 3 stages and advancing the photograph multi-willpower forte estimation strategy in view of the speculation of QWT. Ming Yin, et. Al., clarified about Quaternion Wavelet Analysis for Image Denoising [7].The principle point is the translation and coding of the QWT stage. The Quaternion Wavelet Transform (QWT) is an orthogonal 2D channel bank investigation for grayscale pictures. It gives a quaternion scale space examination, in light of basic work by Bulow [13]. Bulow demonstrated that unpredictable polynomial math $\mathrm{C}$ is ideal for taking care of $1 \mathrm{D}$ flag and that $2 \mathrm{D}$ signals are best portrayed by inserting signal preparing apparatuses in the more broad quaternion variable based math $\mathrm{H}$. Though DWT coefficients are genuine QWT is quaternion esteemed i.e. 4-vectors made of one size and a 3-edge stage. In this manner the data is better isolated to portray all the more unequivocally the picture content.

\section{QUATERnion AnAlytical Signal}

\subsection{Hilbert Transform Concept}

Set $f\left(x, y \in L^{2}\left(R^{2}\right)\right.$, then $f(x, y)$ along then $x$-axis and the $y$-axis and along the $x, y$ axis of the Hilbert transform $f_{H x}(x, y), f_{H y}(x, y)$, and $f_{H x y}(x, y)$, respectively:

$$
f_{H x}(x, y)=\frac{1}{\pi} \int_{R} \frac{f\left(\xi_{1}, y\right)}{x-\xi_{1}} d \xi_{1},
$$


The frequency area with respect to $x$-axis is $f_{H x}(u, v)=-j \sin (u) f(u, v)$

$$
f_{H y}(x, y)=\frac{1}{\pi} \int_{R} \frac{f\left(x, \xi_{2}\right)}{y-\xi_{2}} d \xi_{2},
$$

The frequency area with respect to $y$-axis is $f_{H y}(u, v)=-j \operatorname{sgn}(v) f(u, v)$

$f_{H x y}(x, y)=\frac{1}{\pi^{2}} \iint_{R^{2}} \frac{f\left(\xi_{1}, \xi_{2}\right)}{\left(x-\xi_{1}\right)\left(y-\xi_{2}\right)} d \xi_{1} d \xi_{2}$

The frequency area with respect to $x$-axis and $y$-axis is $f_{H x y}(x, y)=-\operatorname{sgn}(u) \operatorname{sgn}(v) f(u, v)$

\subsection{Quaternion Analytical Signal}

W. R. Hamilton proposed the quaternion in 1843; a feature of quaternions is that multiplication of two quaternions is non-commutative. Hamilton described a quaternion as the quotient of directed lines in a 3dimensional area or equivalently as the quotient of two vectors, and it may be appeared as a completely unique Clifford algebra.

Set

$H=\left\{q=q_{r}+i q_{i}+j q_{j}+k q_{k} \mid q_{r}, q_{i}, q_{j}, q_{k} \in R\right\}$,

Set Three imaginary i, j, k, satisfy the following rules

$i^{2}=j^{2}=k^{2}=-1, i j=-j i=k, j k=-k j=i, k i=-i k=j$. and ountline $q$ as the quaternion.

\section{QUATERnion WAVELET TrANSFORM}

This section completely concentrates on wavelet and scale functions $[5,6]$ of quaternion along with analysis and synthesis structures for single level.

\subsection{Quaternion Wavelet Transform's Structure}

The above talk shows that QWT thru the usage of four actual DWTs, the actual part of QWT is not anything but the very first a part of discrete wavelet and the very last three imaginary elements of the QWT is fashioned thru the Hilbert rework of the number one actual discrete wavelet respectively. Quaternion scale function and wavelet feature's corresponding real additives of QWT are shown inside the following matrix

$$
G=\left(\begin{array}{llll}
\varphi_{h}(x) \varphi_{h}(y) & \varphi_{h}(x) \psi_{h}(y) & \psi_{h}(x) \varphi_{h}(y) & \psi_{h}(x) \psi_{h}(y) \\
\varphi_{g}(x) \varphi_{h}(y) & \varphi_{g}(x) \psi_{h}(y) & \psi_{g}(x) \varphi_{h}(y) & \psi_{g}(x) \psi_{h}(y) \\
\varphi_{h}(x) \varphi_{g}(y) & \varphi_{h}(x) \psi_{g}(y) & \psi_{h}(x) \varphi_{g}(y) & \psi_{h}(x) \psi_{g}(y) \\
\varphi_{g}(x) \varphi_{g}(y) & \varphi_{g}(x) \psi_{g}(y) & \psi_{g}(x) \varphi_{g}(y) & \psi_{g}(x) \psi_{g}(y)
\end{array}\right)
$$

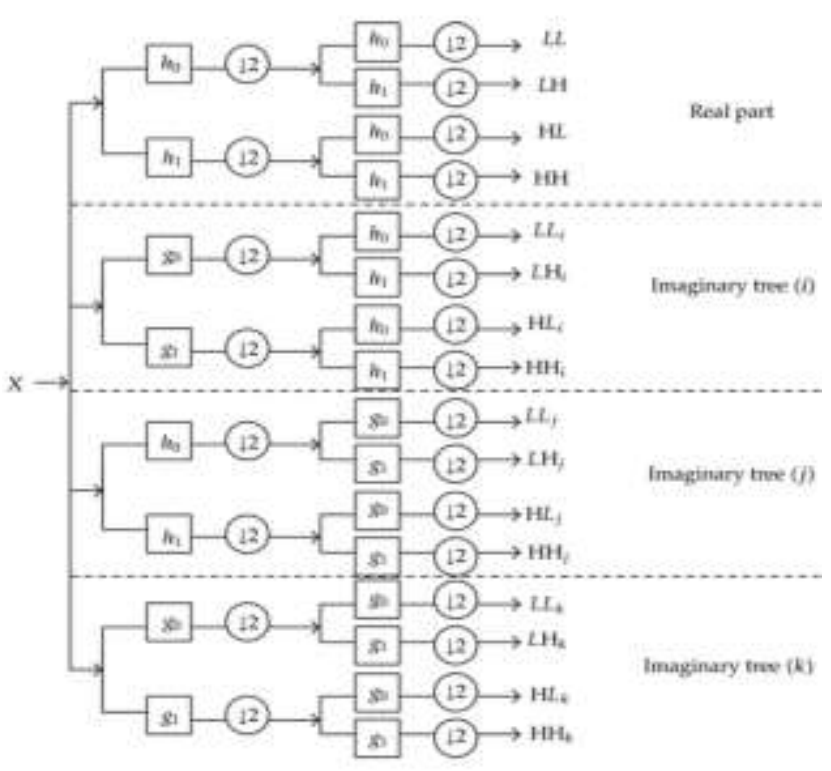

(a) Decomposition Structure

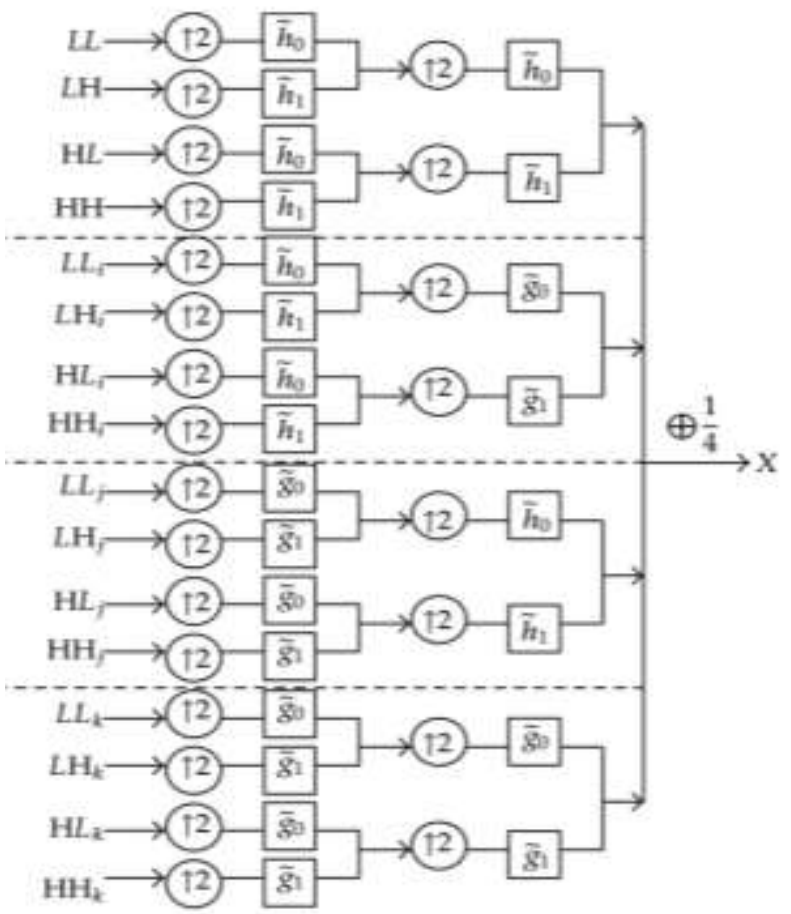

(b) Reconstructionl Structure

Fig1. QWT Analysis \& Synthesis Filter Banks

Figure 1 shows the analysis and synthesis of QWT.

\subsection{The QWT Representation of Image}

The coefficients corresponding to quaternion wavelet transform can constitute a matrix Q :

$$
Q=\left(\begin{array}{llll}
L L_{\varphi_{h}(x) \varphi_{h}(y)} & L H_{\varphi_{h}(x) \psi_{h}(y)} & H L_{\psi_{h}(x) \varphi_{h}(y)} & H H_{\psi_{h}(x) \psi_{h}(y)} \\
L L_{\varphi_{g}(x) \varphi_{h}(y)} & L H_{\varphi_{g}(x) \psi_{h}(y)} & H L_{\psi_{g}(x) \varphi_{h}(y)} & H H_{\psi_{g}(x) \psi_{h}(y)} \\
L L_{\varphi_{h}(x) \varphi_{g}(y)} & L H_{\varphi_{h}(x) \psi_{g}(y)} & H L_{\psi_{h}(x) \varphi_{g}(y)} & H H_{\psi_{h}(x) \psi_{g}(y)} \\
L L_{\varphi_{g}(x) \varphi_{g}(y)} & L H_{\varphi_{g}(x) \psi_{g}(y)} & H L_{\psi_{g}(x) \varphi_{g}(y)} & H H_{\psi_{g}(x) \psi_{g}(y)}
\end{array}\right)
$$

Each column of matrix $\mathrm{Q}$ corresponds to a ' $\mathrm{q}$ '. 


$$
\begin{aligned}
& q 1=L L_{\varphi_{h}(x) \varphi_{h}(y)}+L H_{\varphi_{h}(x) \psi_{h}(y)}+H L_{\psi_{h}(x) \varphi_{h}(y)}+H H_{\psi_{h}(x) \psi_{h}(y)} \\
& q 2=L L_{\varphi_{g}(x) \varphi_{h}(y)}+L H_{\varphi_{g}(x) \psi_{h}(y)}+H L_{\psi_{g}(x) \varphi_{h}(y)}+H H_{\psi_{g}(x) \psi_{h}(y)} \\
& q 3=L L_{\varphi_{h}(x) \varphi_{g}(y)}+L H_{\varphi_{h}(x) \psi_{g}(y)}+H L_{\psi_{h}(x) \varphi_{g}(y)}+H H_{\psi_{h}(x) \psi_{g}(y)} \\
& q 4=L L_{\varphi_{g}(x) \varphi_{g}(y)}+L H_{\varphi_{g}(x) \psi_{g}(y)}+H L_{\psi_{g}(x) \varphi_{g}(y)}+H H_{\psi_{g}(x) \psi_{g}(y)}
\end{aligned}
$$

The signal segment information displays critical local functions; consistent with the idea of quaternion $q=|q| e^{j \varphi} e^{j \theta} e^{j \psi}$,

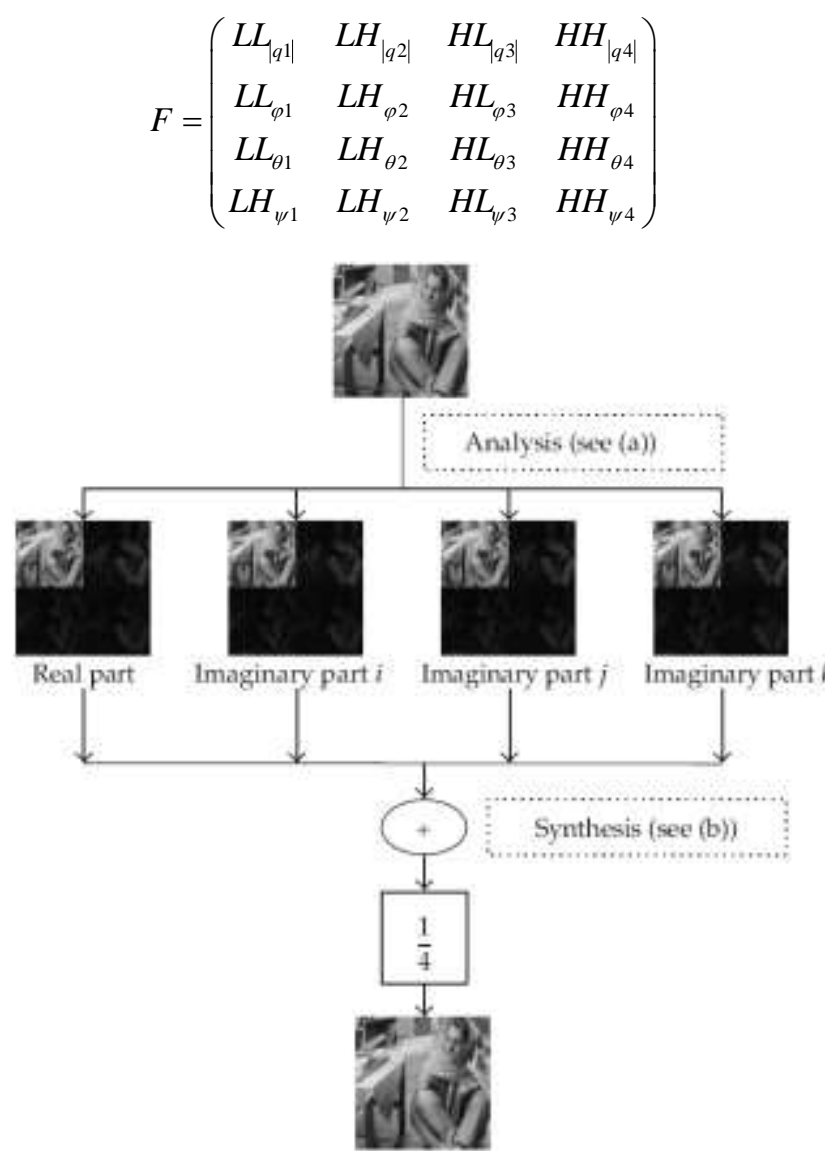

(c) One level OWT decomposition on the Barbara imasc Fig2. The Analysis and Synthesis of quaternion wavelet. Assume that the dimensions of particular image is $m \times n$ and the size of significance and section matrix $\mathrm{F}$ is $\left(2^{2-j} m\right) \times\left(2^{2-j} n\right)$ in the jth scale QWT decomposition.

\subsection{Quaternion Wavelet Transform Filters Design}

Keeping in mind the forestall reason to determine the coefficients of QWT, looking from the shape, the quaternion wavelet channels' framework is like double tree complex wavelet, quaternion wavelet channels' coefficients is quaternion, and it's miles recounted thru utilising the double tree calculation, using an explanatory quaternion wavelet bases at the manner to meet the Hilbert trade. Quaternion wavelet channels are double tree channels, every channel's subtree element includes 2 research channels and more than one amalgamation channels, for my part. In this paper, we make use of the quaternion wavelet with near symmetric orthogonal "Farrs" channels [15] at diploma 1 and Q-bypass double tree channels [16] at extra accelerated degrees.

\section{RESULTS}

In order to assess the proposed set of rules, $512 \times 512$ clinical images were taken and are validated in determine 3 and moreover calculated the evaluation parameters like Compression Ratio (CR), PSNR and MSE for DWT, CWT \& QWT.

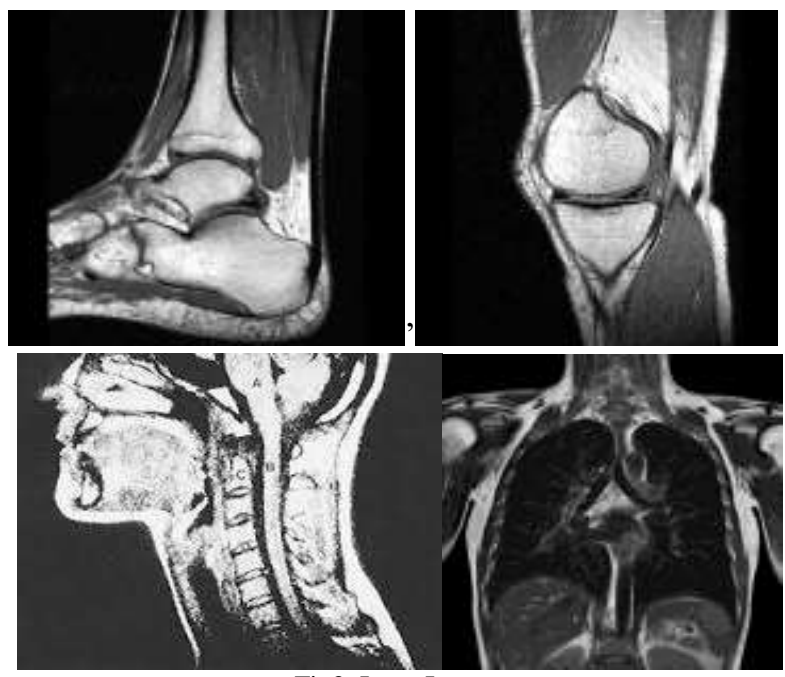

Fig3. Input Images

Evaluation parameters like compression ratio, Peak Signal to Noise Ratio and Mean Square Error are given in the following table along with required graphs.

\begin{tabular}{|c|c|c|c|c|}
\hline $\begin{array}{c}\text { Name } \\
\text { of the } \\
\text { Image }\end{array}$ & $\begin{array}{c}\text { Method } \\
\text { Used }\end{array}$ & PSNR[dB] & CR & MSE \\
\hline \multirow{3}{*}{$\begin{array}{c}\text { MRI } \\
\text { Ankle }\end{array}$} & DWT & 45.1057 & 1.5573 & 2.0663 \\
\cline { 2 - 5 } & CWT & 52.2539 & 2.0646 & 0.3905 \\
\hline \multirow{3}{*}{$\begin{array}{c}\text { MRI } \\
\text { Knee }\end{array}$} & QWT & 63.8152 & 22.6739 & $\begin{array}{c}6.8061 \mathrm{e}- \\
2\end{array}$ \\
\cline { 2 - 5 } & QWT & 30.2430 & 0.8063 & 9.11633 \\
\hline \multirow{3}{*}{$\begin{array}{c}\text { MRI } \\
\text { Neck }\end{array}$} & DWT & 29.9728 & 1.0245 & 2.88151 \\
\cline { 2 - 5 } & QWT & 34.3980 & 1.0495 & 2.78123 \\
\hline \multirow{3}{*}{$\begin{array}{c}\text { MRI } \\
\text { Chest }\end{array}$} & DWT & 41.1716 & 1.3693 & 5.26737 \\
\cline { 2 - 5 } & CWT & 51.0739 & 2.0132 & 0.5128 \\
\hline & QWT & 66.6602 & 23.7151 & $\begin{array}{c}3.5351 \mathrm{e}- \\
2\end{array}$ \\
\hline
\end{tabular}

Table 1: Comparison of evaluation parameters 


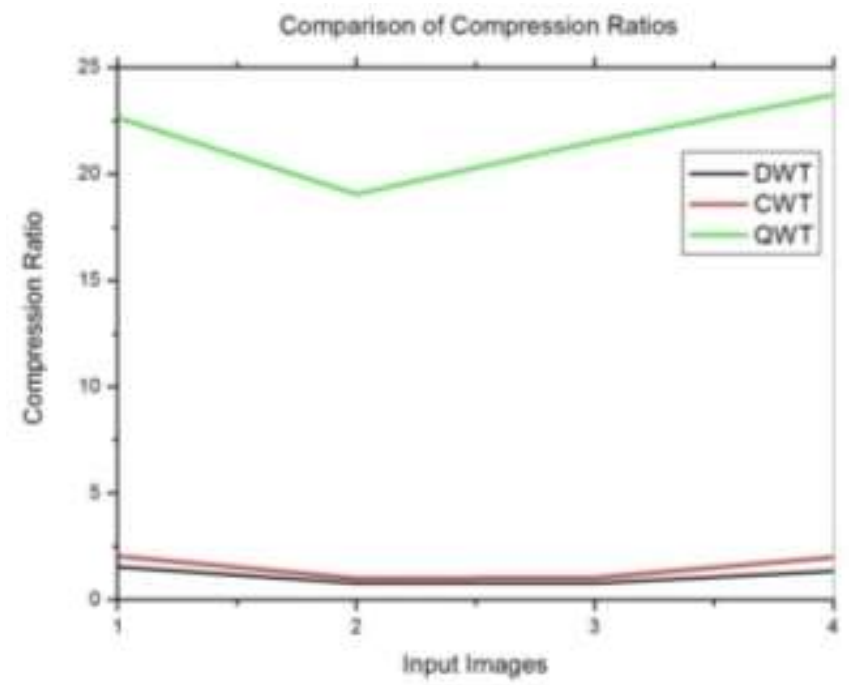

Fig 4. Comparison of Compression ratios [CR]

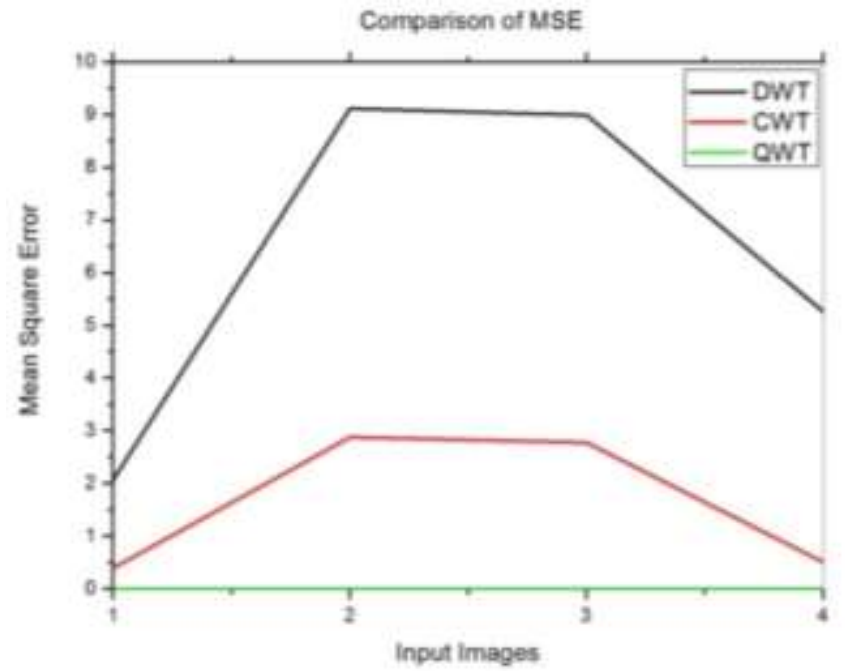

Fig 6. Comparison of MSE

From the above tabular and graphical illustration it's miles clean that the proposed technique (i.e., Compression the usage of Quaternion Wavelet Transform, (QWT) is producing better outcomes in comparison to conventional methods.

\section{Conclusion}

In this paper, Medical Image compression, the use of quaternion wavelets is presented. The proposed approach of compressing the pictures, i.e, quaternion wavelet based compression method consequences in substantial discount in CR and better execution time of the Medical picture without an awful lot lack of information. The number one intention of this paper is to lessen the dimensions of the scientific snap shots. Our studies on this is targeted to reduce the execution time on compressing and provide a excessive safety to the virtual statistics. From the evaluation, it's miles observed that the proposed algorithm will offer higher signal to ratio. From desk, it is found that the higher compression ratio is received for the proposed set of rules at the side of the peak signal to noise ratio.
References:

1. I. W. Selesnick, "Wavelets, a modern tool for signal processing", Physics Today. 60(10):78-79, October 2007.

2. I. W. Selesnick, R. G. Baraniuk, and N. Kingsbury, "The dual-tree complex wavelet rework - A coherent framework for multiscale sign and photograph processing", IEEE Signal Processing Magazine, 22(6):123-151, November 2005.

3. W. L. Chan, H. Choi, and R. G. Baraniuk, "Coherent Multiscale Image Processing the use of Dual-Tree Quaternion Wavelets", in IEEE Transactions on Image Processing, Vol.17, No.7, July 2008.

4. E. B. Corrochano, "Multi-resolution image evaluation the usage of the quaternion wavelet remodel," Numerical Algorithms, vol. 39, no. 1three, pp. 35-55, 2005.

5. E. B. Corrochano, "The concept and use of the quaternion wavelet remodel," Journal of Mathematical Imaging and Vision, vol. 24, no. 1, pp. 19-35, 2006.

6. W. L. Chan, H. Choi, and R. G. Baraniuk, "Coherent multiscale picture processing the use of dual-tree quaternion wavelets," IEEE Transactions on Image Processing, vol. 17, no. 7, pp. 10691082, 2008.

7. Ming Yin, Wei Liu, Jun Shui, and Jiangmin Wu "Quaternion Wavelet Analysis and Application in Image Denoising", Mathematical Problems in Engineering, Volume 2012 (2012), Article ID 493976, 21 pages

8. M. Mitrea, Clifford Waveletes, Singular Integrals and Hardy Spaces, vol. 1575 of Lecture Notes in Mathematics, Springer, 1994.

9. L. Traversoni, "Image evaluation the use of quaternion wavelets," in Geometric Algebra with Applications in Science and Engineering, E. B. C. Corrochano and G. Sobczyk, Eds., pp. 326-343, Birkhäuser, 2001.

10. J. X. He and B. Yu, "Continuous wavelet transforms on the distance L2(R,H;dx)," Applied Mathematics Letters, vol. 17, no. 1, pp. 111-121, 2004. 
11. M. Bahri, "Construction of quaternion-valued wavelets," Matematika, vol. 26, no. 1, pp. 107-114, 2010 .

12. M. Bahri, R. Ashino, and R. Vaillancourt, "Twodimensional quaternion wavelet remodel," Applied Mathematics and Computation, vol. 218, no. 1, pp. 10-21, 2011.

13. T. Bulow, Hypercomplex spectral sign representations for the processing and evaluation of snap shots [Ph.D. Thesis], Christian Albrechts University, Kiel, Germany, 1999.

14. S. G. Mallat, A Wavelet Tour of Signal Processing, Academic Press, San Diego, Calif, USA, 2nd version, 1999.

15. A. F. Abdelnour and I. W. Selesnick, "Nearly symmetric orthogonal wavelet bases," in Proceedings of the IEEE Interntional Conference on Acoustics, Speech, and Signal Processing (ICASSP '01), May 2001.

16. N. Kingsbury, "Complex wavelets for shift invariant analysis and filtering of signals," Applied and Computational Harmonic Analysis, vol. 10, no. Three, pp. 234-253, 2001. 